\title{
Impact of Hospital Policies on Breastfeeding Outcomes
}

\author{
Kenneth D. Rosenberg, ${ }^{1,2}$ John D. Stull, ${ }^{1,2}$ Michelle R. Adler, ${ }^{1,3}$ Laurin J. Kasehagen, ${ }^{1}$ \\ and Andrea Crivelli-Kovach ${ }^{4}$
}

\begin{abstract}
Objective: The objective of this study was to explore the association between the Ten Steps of the Baby Friendly Hospital Initiative (BFHI) of the World Health Organization (Geneva, Switzerland) and breastfeeding at 2 days and 2 weeks.

Methods: A 65-question institutional survey assessing compliance with the Ten Steps was used to determine an overall breastfeeding Support Score for each of Oregon's 57 birthing hospitals. Hospital breastfeeding outcomes were obtained from the newborn metabolic screening forms.

Results: Hospitals' overall breastfeeding Support Scores ranged from 49.4 to 98.2 out of a possible total score of 100. Hospital compliance with individual Steps ranged from 5.3\% for Step 2 (staff training) to 93\% for Step 4 (helping with breastfeeding initiation) and Step 8 (encouraging feeding on demand). After controlling for institutional differences (by multivariate linear regression) we found that increases in overall hospital breastfeeding Support Scores were associated with increases in breastfeeding percentage at 2 days $(p=0.021)$ and at 2 weeks postpartum $(p=0.011)$. In analyzing each Step individually, however, only the presence of a written hospital policy was independently associated with breastfeeding percent $(p=0.028)$.

Conclusions: This institutional-level evaluation corroborates previous findings demonstrating that increased implementation of the Ten Steps is associated with increased breastfeeding. Further, it suggests that hospitals with comprehensive breastfeeding policies are likely to have better breastfeeding support services and better breastfeeding outcomes. Hospitals may consider using these results to prioritize breastfeeding support services through development of hospital breastfeeding policies and to utilize institutional surveys as a component of breastfeeding quality improvement initiatives.
\end{abstract}

\section{Introduction}

B REASTFEEDING HAS BEEN SHOWN to provide multiple health benefits for both infants and mothers. ${ }^{1,2}$ Compared to formula-fed infants, breastfed infants have fewer middle ear infections, ${ }^{3,4}$ decreased rates of diarrhea, ${ }^{5}$ a reduced risk of severe respiratory tract infections, ${ }^{6}$ and decreased childhood asthma. ${ }^{7}$ Mothers who breastfeed have a decreased risk of ovarian and breast cancers 8,9 and may return to baseline weight more rapidly following delivery. ${ }^{10}$ Despite these benefits, a 2006 breastfeeding surveillance study by the Centers for Disease Control and Prevention (CDC) (Atlanta, GA) of women giving birth in 2004 showed that nationwide only $74 \%$ of women initiated breastfeeding, and only $11 \%$ exclusively breastfed through 6 months. ${ }^{11}$

In 1991, recognizing the importance of increasing breastfeeding worldwide, the World Health Organization (WHO)
(Geneva, Switzerland), in collaboration with the United Nation's Children's Fund (UNICEF) (New York, NY), launched the Baby Friendly Hospital Initiative (BFHI). This initiative promotes breastfeeding within the hospital setting through the implementation of Ten Steps (Table 1). The proportion of infants initiating breastfeeding improves after hospitals implement the BFHI practices. ${ }^{12-15}$ Despite the well-studied benefit of providing BFHI support services and the U.S. Department of Health and Human Services' recommendation to implement these practices, ${ }^{2}$ U.S. hospitals have been slow to adopt the policies and practices outlined in the BFHI. ${ }^{16}$ As of January 2008, only 62 hospitals were designated as "Baby Friendly" nationwide, four of which are in Oregon. ${ }^{17}$

The purpose of this study was to determine whether the level of hospital implementation of any or all of the Ten Steps of the $\mathrm{BFHI}$, measured by a modified version of the Crivelli-Kovach tool, ${ }^{18-20}$ influences the percentage of women who breastfeed.

${ }^{1}$ Office of Family Health, Oregon Public Health Division, Portland, Oregon.

Departments of ${ }^{2}$ Public Health and Preventive Medicine and ${ }^{3}$ Family Medicine, Oregon Health \& Science University, Portland, Oregon.

${ }^{4}$ Department of Medical Science and Community Health, Arcadia University, Glenside, Pennsylvania. 
Table 1. BFHI: Ten Steps and Dimensions

Baby friendly step

Dimensions of step

(number of questions)

1. Have a written breastfeeding policy that is routinely communicated to all health care staff

2. Train all health care staff in skills necessary to implement the policy

3. Inform all pregnant women about the benefits and management of breastfeeding

4. Help mothers initiate breastfeeding within 30 minutes of birth

5. Show mothers how to breastfeed, and how to maintain lactation even if separated from their infants

6. Give newborn infants no food or drink other than breastmilk, unless medically indicated

7. Practice rooming-in: allow mothers and infants to remain together 24 hours/day

8. Encourage breastfeeding on demand

9. Give no artificial teats or pacifiers to breastfed infants

10. Foster the establishment of breastfeeding support groups and refer mothers to them on discharge from the hospital
Elements of policy (10)

Hospital staff knowledge of policy (2)

Hospital staff practice of policy (2)

Accessibility of policy (1)

Evaluation of hospital breastfeeding practices (1)

Breastfeeding training program coordinator (1)

Basic breastfeeding training offered (2)

Ongoing breastfeeding in-services (1)

Specifically designated breastfeeding support staff member (2)

Maternal plans for infant feeding (2)

Prenatal breastfeeding classes/information sessions (1)

Postpartum provision of breastfeeding information (3)

Time between delivery and first breastfeeding (2)

Assistance with first breastfeeding (1)

Instruction on breastfeeding techniques (3)

Maintaining lactation if newborn is ill (1) ${ }^{\mathrm{a}}$

Expressed breastmilk provided to ill infant $(1)^{\mathrm{b}}$

Routine provision of non-breastmilk supplements (3)

Provision of formula promotional items (2)

Hospital receives free formula (1)

Rooming-in availability (2)

Rooming-in policy (1)

Rooming-in criteria (1)

Rooming-in practiced (2)

Timing of breastfeeding: schedule, on cue, duration (4)

Offered pacifier between feeds (1)

Hospital breastfeeding support post-discharge (2)

Referral to community breastfeeding support (1)

Written breastfeeding information provided at discharge (1)

aDimension only used in hospitals where mothers of ill infants stay.

bimension only used in hospitals with neonatal intensive care units.

\section{Materials and Methods}

\section{Study design}

Using a cross-sectional design, Oregon birthing hospitals were surveyed about their breastfeeding support practices. Responses were linked with the proportion of infants, born in that hospital during the same year, who were reported to have been breastfed on 2-day and 2-week Newborn Metabolic Screening (NBS) forms.

\section{Data collection}

In order to facilitate the evaluation of the implementation of BFHI practices at an institutional level, one of the authors (A.C.-K.) developed a survey that can be completed by hospital administrators. Responses can be easily converted into a score that indicates the level of breastfeeding support provided by the institution. ${ }^{18}$

A 65-question survey instrument, modeled after one used previously, ${ }^{18-20}$ was developed to evaluate breastfeeding support in Oregon's 57 birthing hospitals. The Crivelli-Kovach methodology had been reviewed for content validity by a committee of breastfeeding experts in maternal and child health, including physicians, nurses, lactation consultants, and doctorally prepared researchers, after having been pilot-tested in four hospitals in Delaware and New Jersey. Our most important adaptation was mode of administration: the Crivelli-Kovach methodology included face-to-face interviews with groups of three to eight people completing one questionnaire per hospital; we did phone interviews with only one person per hospital.

Our survey asked the respondent to provide basic hospital demographic information including location, bed capacity, number of deliveries in the last year, average annual number of deliveries, and nursery level. The remainder of the questionnaire asked for detailed information regarding hospital breastfeeding support services. Retaining the Pennsylvania survey format, questions were organized into sections that correspond to the Ten Steps. Each step consists of one to four dimensions, and each dimension is assessed using one to four questions (Table 1). Changes made to the original survey were based on recommendations of the 1998 Oregon Breastfeeding Task Force. Several nursing personnel who worked in hospital nurseries or obstetrical units outside of Oregon reviewed and piloted the final version. 
During September and October 1998, all Oregon birthing hospitals were called by the researcher (J.D.S.) to identify a respondent (either a nurse manager or a lactation specialist), and survey questions were faxed to all respondents. Thirtyseven percent of respondents were interviewed by phone after they had received the questions by fax. The remaining $63 \%$ of respondents completed the survey in writing, faxed replies back to the researcher, and were subsequently called by the researcher to discuss and confirm answers. Interviews were completed at each of Oregon's 57 birthing hospitals.

Data on breastfeeding at 2 days and 2 weeks were obtained from the 1997 Oregon NBS program. The screening form identifies the delivery hospital as well as information on all food sources from which the infant received nutrition in the 24 hours prior to the test. Individual infant feeding information was aggregated for each hospital. The breastfeeding information obtained at the first NBS is identified as "2-day" data and is a proxy for breastfeeding initiation. The information from the second NBS is identified as "2-week" breastfeeding data and is a proxy for breastfeeding continuation.

\section{Data analysis}

Hospital breastfeeding support was quantified by creating an overall composite hospital breastfeeding Support Score. An average score for each of the Ten Steps was calculated based on the number of questions for the individual step. Each average was converted into an individual Step Score between 0 and 10. These individual Step Scores were used to determine the level of compliance with each of the individual steps. A hospital was defined as "highly compliant" with a particular step if it scored 8.0-10, "moderately compliant" if it scored 4.0-7.9, and "poorly compliant" if it scored 0-3.9. The 10 individual Step Scores were summed to obtain the final composite Support Score out of a possible 100 points. Hospitals were categorized as being "poorly compliant" (less than 60.0), "moderately compliant" (60.0-79.9), or "highly compliant" (80.0 or greater).

Two-day and 2-week breastfeeding prevalences were obtained for each Oregon birthing hospital from the 1997 NBS database. Infants were considered to have been breastfed if the NBS form indicated that they had received breastmilk in the 24 hours prior to each of the tests. The 2-day data (often collected when the infant is less than 2 days old) are collected by hospitals at the time of the heel-sticks. The 2-week data are collected by pediatricians and other infant providers, usually at the first pediatric visit. Both are reported to the Oregon Public Health Laboratory.

Data analysis was conducted using Stata Statistical Software, Release $6{ }^{21}$ SPSS for Windows, Release 9.0.0, ${ }^{22}$ and SAS, Version 9.1.3. ${ }^{23}$ Frequencies were calculated for hospital demographic characteristics (location, hospital size, annual number of deliveries, and nursery level), for the level of implementation of each of the Ten Steps, and for overall hospital breastfeeding support. To explore whether the demographic characteristics of hospitals with high 2-week breastfeeding levels were different from hospitals with low 2-week breastfeeding levels, $\chi^{2}$ tests were used. Finally, linear regression modeling was conducted to determine whether breastfeeding was associated with the level of compliance with any of the individual Ten Steps and/or with overall breastfeeding support services. Multivariate models for individual Step evaluation included all demographic variables available. As expected, a correlation matrix showed a high degree of collinearity between many of the individual Steps. Consequently, it was decided to retain any individual Step in the final multivariate model that had a bivariate significance of $p<0.15$. Final analysis inferred statistical significance at $p<0.05$ for each Step.

\section{Results}

\section{Hospital demographics}

All (100\%) of the 57 Oregon birthing hospitals responded to the survey. Most were located in rural areas or in small towns $(n=37,64.9 \%)$, had level I nurseries $(n=37,64.9 \%)$, and had less than 100 beds $(n=32,56.1 \%)$. The number of deliveries ranged from 84 to 8,933 with the majority $(n=38$, $66.7 \%$ ) having $\geq 400$ deliveries in 1997 (Table 2 ). The $\chi^{2}$ analysis showed that hospitals with higher breastfeeding percentages at initiation and 2 weeks were not statistically different from hospitals with lower breastfeeding percentages with regard to location, nursery level, number of births, or bed capacity (data not shown).

\section{Hospital breastfeeding support}

Table 3 shows hospital compliance with the Ten Steps. Overall, out of a total of 100 points, Support Scores ranged from a low of 49.4 to a high of $98.2($ mean $=73.4, \mathrm{SD}=11.4)$. Of the 57 birthing hospitals in Oregon, six (10.5\%) were poorly compliant (Support Score <60), 36 (63.2\%) were moderately compliant (Support Score 60.0-79.9), and 15 (26.3\%) were highly compliant (Support Score $\geq 80.0$ ). In general, Oregon hospitals excelled at assisting with breastfeeding initiation (Step 4), encouraging demand feeding (Step 8), and providing breastfeeding information (Step 3). The least implemented steps were providing staff with breastfeeding training (Step 2), avoiding supplementation (Step 6), and having and communicating a written breastfeeding policy (Step 1).

Table 2. Demographic Characteristics of Hospitals of Oregon's 57 Birthing Hospitals

\begin{tabular}{llr}
\hline Characteristic & \multicolumn{1}{c}{ Subcategory } & Number (\%) \\
\hline Location & Urban & $12(21.1)$ \\
& Suburban & $8(14.0)$ \\
& Small town & $13(22.8)$ \\
Nursery level & Rural & $24(42.1)$ \\
& Level I (basic) & $37(64.9)$ \\
& Level II (specialty care) & $14(24.6)$ \\
Births (1997) & Level III (NICU) & $6(10.5)$ \\
& $<399$ & $19(33.3)$ \\
& $400-699$ & $9(15.8)$ \\
& $700-1,499$ & $11(19.3)$ \\
& $1,500-2,999$ & $12(21.1)$ \\
Bed capacity & $\geq 3,000$ & $6(10.5)$ \\
& $<30$ & $8(14.0)$ \\
& $30-99$ & $24(42.1)$ \\
& $100-299$ & $11(19.3)$ \\
& $\geq 300$ & $8(14.0)$ \\
& Missing & $6(10.5)$ \\
\hline
\end{tabular}

NICU, neonatal intensive care unit. 


\section{Relationship between hospital breastfeeding support and breastfeeding}

We found a positive association between hospitals' overall breastfeeding Support Scores and breastfeeding. The association became stronger and statistically significant after controlling for hospital location (urban vs. rural), size, number of deliveries, and nursery level at 2 days $\left(R^{2}=0.247, p=\right.$ $0.021)$ and at 2 weeks $\left(R^{2}=0.183, p=0.011\right.$ ) (data not shown). In order to evaluate whether one or more specific elements of the overall support were particularly beneficial, each Step was analyzed individually using linear regression (Table 4). While staff training and pacifier use were associated with breastfeeding at 2 days in the bivariate analysis $(p<0.050)$, these associations were not found in multivariate analysis. Having a hospital policy (Step 1) and training staff (Step 2) were each associated with breastfeeding at 2 weeks in bivariate analysis. When these factors were placed in a multivariate model, however, only having a written breastfeeding policy that was communicated to staff (Step 1) remained associated with breastfeeding at 2 weeks $(p=$ 0.028).

\section{Discussion}

We found that comprehensively implementing the BFHI Ten Steps promotes breastfeeding in the infant's first 2 weeks. Further, this study demonstrates that hospitals that administratively support breastfeeding through written hospital breastfeeding policies provide better breastfeeding support services that lead to better breastfeeding outcomes.

\section{Benefits of the BFHI}

The synergistic nature of multiple breastfeeding support interventions found in this study is supported by previous findings. DiGirolamo et al., ${ }^{24}$ for example, found that addressing multiple BFHI Steps simultaneously is more effective than promoting any one Step in particular. They surveyed 1,085 postpartum women with prenatal intention to breastfeed regarding which of five Baby Friendly Steps they experienced in the hospital. The more Steps that a woman experienced, the more likely she was to still be breastfeeding at 6 weeks postpartum. Additionally, a trial in Belarus following over 17,000 postpartum patients for 1 year revealed that those women who gave birth in Baby Friendly hospitals were more likely to still be breastfeeding at 3, 6, and 12 months as compared to women who gave birth in non-Baby Friendly hospitals. ${ }^{15}$ Based on these and other studies, the Canadian Task Force on Preventive Health strongly recommends that maternity hospitals implement at least four Steps of the Baby Friendly Hospital Initiativeearly maternal contact, rooming-in, postpartum support services, and elimination of the provision of commercial discharge packages. ${ }^{25}$ Further, the U.S. Department of Health and Human Services recommends that hospitals implement ". . . practices that promote breastfeeding, such as the 'Ten Steps to Successful Breastfeeding' . . ."2

\section{Evidence to encourage administrative support of breastfeeding}

This study shows that hospitals that have a written policy have better breastfeeding outcomes at 2 weeks. Administra-

Table 3. Number of Hospitals Attaining Specific Level of Breastfeeding Support as Measured by Compliance with the Ten Steps

\begin{tabular}{|c|c|c|c|c|}
\hline & & \multicolumn{3}{|c|}{ Level of overall compliance based on Support Score } \\
\hline & & $\begin{array}{c}\text { Poor } \\
(<60.0) \\
N(\%)\end{array}$ & $\begin{array}{c}\text { Moderate } \\
(60.0-79.9) \\
N(\%)\end{array}$ & $\begin{array}{c}\text { High } \\
(\geq 80.0) \\
N(\%)\end{array}$ \\
\hline & & $6(10.5)$ & $36(63.2)$ & $15(26.3)$ \\
\hline \multirow[b]{2}{*}{ Step } & \multirow[b]{2}{*}{ Description } & \multicolumn{3}{|c|}{$\begin{array}{l}\text { Level of compliance with each Step ranked by } \\
\text { percentage of hospitals with high Step Scores }\end{array}$} \\
\hline & & $\begin{array}{c}\text { Poor } \\
(0-3.9) \\
N(\%)\end{array}$ & $\begin{array}{c}\text { Moderate } \\
(4.0-7.9) \\
N(\%)\end{array}$ & $\begin{array}{c}\text { High } \\
(8.0-10) \\
N(\%)\end{array}$ \\
\hline 4 & Breastfeeding initiation & $0(0)$ & $4(7.0)$ & $53(93.0)$ \\
\hline 8 & Demand feeding & $0(0)$ & $4(7.0)$ & $53(93.0)$ \\
\hline 3 & Breastfeeding information given & $0(0)$ & $19(33.3)$ & $38(66.7)$ \\
\hline 7 & Rooming-in & $0(0)$ & $27(47.4)$ & $30(52.6)$ \\
\hline 5 & Breastfeeding instruction/maintenance & $1(1.8)$ & $28(49.1)$ & $28(49.1)$ \\
\hline 9 & Pacifier use & $3(5.3)$ & $29(50.9)$ & $25(43.9)$ \\
\hline 10 & Discharge plan & $8(14.0)$ & $24(42.1)$ & $25(43.9)$ \\
\hline 1 & Written policy & $17(29.8)$ & $25(43.9)$ & $15(26.3)$ \\
\hline 6 & Supplementation & $4(7.0)$ & $42(73.7)$ & 11 (19.3) \\
\hline 2 & Staff training & $24(42.1)$ & $30(52.6)$ & $3(5.3)$ \\
\hline
\end{tabular}

aThe overall Support Score for a given hospital is the sum of the Step Scores for Steps 1-10 for that hospital. The maximum possible score is 100.

${ }^{\mathrm{b}} \mathrm{A}$ Step Score is an adjusted average of the scores for each dimension within an individual Step. Each individual Step Score ranges from 0 to 10. 
Table 4. Association Between Implementation of Individual Steps and Breastfeeding at 2 Days and 2 WeEKS

\begin{tabular}{|c|c|c|c|c|c|c|c|c|}
\hline \multirow[b]{3}{*}{ Variable } & \multicolumn{4}{|c|}{2 days (initiation) ${ }^{\mathrm{a}}$} & \multicolumn{4}{|c|}{2 weeks ${ }^{\mathrm{a}}$} \\
\hline & \multicolumn{2}{|c|}{ Bivariate } & \multicolumn{2}{|c|}{ Multivariate } & \multicolumn{2}{|c|}{ Bivariate } & \multicolumn{2}{|c|}{ Multivariate } \\
\hline & $\begin{array}{c}B \\
\text { coefficient }\end{array}$ & $\mathrm{v}_{\text {value }}^{\mathrm{p}}$ & $\begin{array}{c}B \\
\text { coefficient }\end{array}$ & $\underset{\text { value }}{\mathrm{p}}$ & $\begin{array}{c}B \\
\text { coefficient }\end{array}$ & $\underset{\text { value }}{\mathrm{p}}$ & $\begin{array}{c}B \\
\text { coefficient }\end{array}$ & $\underset{\text { value }}{\mathrm{p}}$ \\
\hline \multicolumn{9}{|l|}{ Individual step } \\
\hline 1. Written policy & 0.198 & 0.147 & 0.148 & 0.364 & 0.370 & 0.005 & 0.352 & 0.028 \\
\hline 2. Staff training & 0.269 & 0.047 & 0.239 & 0.121 & 0.337 & 0.010 & 0.214 & 0.152 \\
\hline $\begin{array}{l}\text { 3. Breastfeeding information } \\
\text { given }\end{array}$ & 0.139 & 0.313 & & & 0.171 & 0.202 & & \\
\hline 4. Breastfeeding initiation & 0.026 & 0.849 & & & 0.129 & 0.337 & & \\
\hline 5. Breastfeeding instruction & -0.019 & 0.891 & & & -0.037 & 0.787 & & \\
\hline 6. Supplementation & 0.134 & 0.328 & & & 0.187 & 0.164 & & \\
\hline 7. Rooming-in & -0.006 & 0.963 & & & 0.189 & 0.158 & & \\
\hline 8. Demand feeding & 0.064 & 0.640 & & & -0.015 & 0.914 & & \\
\hline 9. Pacifier use & 0.266 & 0.049 & 0.165 & 0.233 & 0.094 & 0.488 & & \\
\hline 10. Discharge plan & -0.034 & 0.807 & & & 0.050 & 0.714 & & \\
\hline \multicolumn{9}{|l|}{ Demographics } \\
\hline Beds & -0.303 & 0.034 & -0.242 & 0.434 & -0.127 & 0.375 & -0.266 & 0.390 \\
\hline Births in 1997 & -0.293 & 0.030 & -0.157 & 0.555 & 0.059 & 0.663 & 0.127 & 0.605 \\
\hline Urban versus rural & 0.335 & 0.013 & 0.166 & 0.404 & 0.174 & 0.196 & 0.172 & 0.359 \\
\hline Nursery level & -0.085 & 0.536 & 0.222 & 0.222 & -0.085 & 0.530 & 0.040 & 0.827 \\
\hline
\end{tabular}

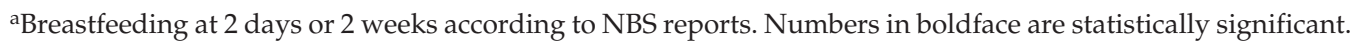

tive prioritization of breastfeeding support drives the hospital practices that lead to improved breastfeeding. Although the institutional survey tools varied in each of the following studies, it is noteworthy that $70 \%$ of hospitals in Oregon had a breastfeeding policy compared to $62 \%$ in Minnesota, $28 \%$ in Missouri, and $2.4 \%$ in North Dakota. ${ }^{26}$ The widespread institutional support for breastfeeding support services may be one of the reasons that Oregon consistently has a higher proportion of breastfeeding mothers than other states. ${ }^{11}$

While hospital policy influences 2-week breastfeeding outcomes, it does not significantly affect breastfeeding initiation in this study $(p=0.364)$. A variety of explanations for this finding are plausible. First, maternal breastfeeding intention (before delivery) is an important part of initiation; its impact probably decreases over time. ${ }^{27,28}$ Second, $93 \%$ of hospitals report always assisting with breastfeeding initiation, so 2day feeding activities may reflect the almost universal practice of assistance with initiation as opposed to the overall hospital policy.The Division of Nutrition, Physical Activity, and Obesity of the CDC conducted a new Maternity Practices in Infant Nutrition and Care Survey (mPINC) in late 2007 with plans to repeat the survey every other year. (Two of us [K.D.R. and A.C.-K.] were members of a group that advised CDC on the development of the survey.) The survey is modeled after Crivelli-Kovach's survey ${ }^{18}$ except that it will be conducted entirely by mail or internet. The goal of the mPINC is to assess maternity care practices-including changes over time. It is expected that the mPINC will generate pressure for hospitals to improve their practices around breastfeeding in the newborn hospitalization, especially since individual benchmark reports will be sent back to each hospital. Our study shows that a modified version of Crivelli-Kovach's survey ${ }^{18}$ can generate useful estimates of hospital practice around breastfeeding in the newborn hospi- talization. Despite some changes in methodology between our survey and the mPINC, we expect that we will be able to assess changes in hospital practices in Oregon by comparing the results of the two surveys.

\section{Limitations}

Inherent in this institutional-level study are some important limitations. Because the survey data were self-reported by a single person at each hospital, response bias was unavoidable. Overall the direction and magnitude of the bias are difficult to predict and would likely have resulted in nondifferential misclassification, thereby potentially underestimating the strength of the association found. Somewhat more problematic, however, are the individual responses as they relate to the relationship between hospital policy and practice. Survey respondents may have been involved with the creation and/or implementation of hospital breastfeeding policies, and consequently may have reported results more consistent with the policy than with the actual practice of the maternity care staff. This potential over-reporting of breastfeeding practices in hospitals with breastfeeding policies may have contributed to the finding that hospitals with breastfeeding policies have better breastfeeding Support Scores. For future studies, both the response and selection biases could be significantly reduced if responses were requested from a variety of individuals in different positions within the hospital.

A second set of limitations comes from the breastfeeding data from the NBS program. The "2-day" data are most often collected 12-36 hours after birth. These data may reflect maternal intention to breastfeed as much as initiation of breastfeeding. The advantage of these data is that they are recorded for almost every newborn. The "2-week" breast- 
feeding data are generally collected at the first pediatric visit. Fewer infants get the "2-week" screen than the "2-day" screen, and breastfeeding information is not uniformly recorded. Because of its timing, it is probably a better proxy for continued breastfeeding than the "2-day" data.

While this study was able to control for hospital size, location (rural vs. urban), number of deliveries, and level of nursery, other potential institutional-level confounders such as cesarean section rate, the ethnic demographic, and the socioeconomic distribution of the patient population were not available. Other studies have demonstrated that these factors may have an effect on breastfeeding. ${ }^{13,19}$ Further, while individual-level confounders such as income, education, infant feeding intent prior to delivery, parity, and previous breastfeeding experience potentially impact individual breastfeeding decisions, ${ }^{15,24,27}$ these variables are difficult to assess through this type of institutional survey. The possibility that any of these confounders might have had an effect on the results found in this study cannot be excluded.

Finally, compared with breastfeeding data from the Ross Mothers Survey, ${ }^{29}$ the Pregnancy Risk Assessment Monitoring System (PRAMS), ${ }^{30}$ and the National Immunization Survey (NIS), ${ }^{11}$ NBS data have been shown to under-report breastfeeding initiation by about $20 \%$ (K.D.R., unpublished data). Because the NBS cards are completed independently by providers and ancillary staff, bias is likely to be non-differential and should not affect the validity or generalizability of the study results.

Despite the limitations of the design, the fact that the results of this study parallel those of similar studies provides confidence in the validity of using a hospital survey to study breastfeeding support services. Future studies would benefit from including additional questions related to other potential institutional-level confounders, obtaining responses from multiple individuals at each institution, and validating the hospital staff responses with individual patient responses to similar questions.

\section{Conclusions}

\section{Public health implications}

Given the suboptimal breastfeeding rates in many areas of the United States, there is a need for strategies to improve and measure hospital breastfeeding support services. Despite its limitations, this study lends support to the hypothesis that simply having a clear and comprehensive written hospital breastfeeding policy that is communicated to staff promotes better breastfeeding support services that in turn correlate with better breastfeeding outcomes. Further, with minor modifications, a hospital-level survey similar to the one used in this study is an inexpensive and practical tool that can be used to objectively measure these important hospital breastfeeding support services. As part of an ongoing institutional quality improvement process, breastfeeding advocates may consider recommending to administrators the creation of a hospital breastfeeding policy and the monitoring of services using a simple hospital-level survey. On a population level, the results of surveys such as the one used in this study may be used to make evidence-based policy decisions and monitor improvements in breastfeeding support services over time.

\section{Acknowledgments}

The authors thank Alfredo P. Sandoval, M.S., and Liangrong Wu, M.A., MPH, for their assistance with data analysis; Jihong Liu, D.Sc., Thomas Brundage, Ph.D., and Aleksandra Sumic, $\mathrm{MPH}$, for their statistical support; Laurence Grummer-Strawn, Ph.D., for his editorial assistance; and the Oregon Public Health Laboratory for collecting and sharing the feeding information from the Newborn Metabolic Screening program.

\section{References}

1. American Academy of Pediatrics, Work Group on Breastfeeding. Breastfeeding and the use of human milk. Pediatrics 1997;100:1035-1039.

2. US Department of Health and Human Services. HHS Blueprint for Action on Breastfeeding. Office on Women's Health, U.S. Department of Health and Human Services, Washington, DC, 2000.

3. Aniansson G, Alm B, Andersson B, et al. A prospective cohort study on breast-feeding and otitis media in Swedish infants. Pediatr Infect Dis J 1994;13:183-188.

4. Sassen ML, Brand R, Grote JJ. Breast-feeding and acute otitis media. Am J Otolaryngol 1994;15:351-357.

5. Sterling LM, Richardson J, Ellis M. Clinical inquiries. Does breastfeeding protect against viral GI infections in children $<2$ years old? I Fam Pract 2003;52:805-806.

6. Bachrach VR, Schwarz E, Bachrach LR. Breastfeeding and the risk of hospitalization for respiratory disease in infancy: A meta-analysis. Arch Pediatr Adolesc Med 2003;157:237-243.

7. Gdalevich M, Mimouni D, Mimouni M. Breast-feeding and the risk of bronchial asthma in childhood: a systematic review with meta-analysis of prospective studies. L Pediatr 2001;139:261-266.

8. Labbok MH. Effects of breastfeeding on the mother. Pediatr Clin North Am 2001;48:143-158.

9. Collaborative Group on Hormonal Factors in Breast Cancer. Breast cancer and breastfeeding: Collaborative reanalysis of individual data from 47 epidemiological studies in 30 countries, including 50302 women with breast cancer and 96973 women without the disease. Lancet 2002;360:187-195.

10. Fraser AB, Grimes DA. Effect of lactation on maternal body weight: A systematic review. Obstet Gynecol Surv 2003; 58:265-269.

11. Centers for Disease Control and Prevention. Breastfeeding Practices-Results from the National Immunization Survey. http://www.cdc.gov/breastfeeding/data/NIS_data/data_ 2004.htm (accessed May 1, 2008).

12. Cropley L, Herwehe JC. Evaluation of institutional support for breastfeeding among low-income women in the metropolitan New Orleans area. I Am Diet Assoc 2002;102:94-96.

13. Philipp BL, Malone KL, Cimo S, et al. Sustained breastfeeding rates at a US baby-friendly hospital. Pediatrics 2003; 112:e234-e236.

14. Philipp BL, Merewood A, Miller LW, et al. Baby-friendly hospital initiative improves breastfeeding initiation rates in a US hospital setting. Pediatrics 2001;108:677-681.

15. Kramer MS, Chalmers B, Hodnett ED, et al. Promotion of Breastfeeding Intervention Trial (PROBIT): A randomized trial in the Republic of Belarus. IAMA 2001;285:413-420.

16. Philipp BL, Merewood A. The baby-friendly way: The best breastfeeding start. Pediatr Clin North Am 2004;51:761-783.

17. Baby-Friendly USA. US Baby Friendly Hospitals and Birth Centers. http://www.babyfriendlyusa.org/eng/03. html (accessed January 26, 2008). 
18. Kovach AC. An assessment tool for evaluating hospital breastfeeding policies and practices. [H Hum Lact 1996;12:41-45.

19. Kovach AC. A 5-year follow-up study of hospital breastfeeding policies in the Philadelphia area: A comparison with the ten steps. I Hum Lact 2002;18:144-154.

20. Kovach AC. Hospital breastfeeding policies in the Philadelphia area: A comparison with the ten steps to successful breastfeeding. Birth 1997;24:41-48.

21. StataCorp. Stata Statistical Software: Release 6. StataCorp., College Station, TX, 2003.

22. SPSS, Inc. SPSS for Windows, Release 9.0. SPSS, Inc., Chicago, IL, 1998.

23. SAS Institute. SAS/STAT, Version 9.1.3. SAS Institute, Cary, NC, 2006.

24. DiGirolamo AM, Grummer-Strawn LM, Fein S. Maternity care practices: Implications for breastfeeding. Birth 2001;28: 94-100.

25. Palda VA, Guise JM, Wathen CN, et al. Interventions to promote breast-feeding: Applying the evidence in clinical practice. CMAJ 2004;170:976-978.

26. Dodgson JE, Allard-Hale CJ, Bramscher A, et al. Adherence to the ten steps of the Baby-Friendly Hospital Initiative in Minnesota hospitals. Birth 1999;26:239-247.
27. Kuan LW, Britto M, Decolongon J, et al. Health system factors contributing to breastfeeding success. Pediatrics 1999;104:e28.

28. Wright A, Rice S, Wells S. Changing hospital practices to increase the duration of breastfeeding. Pediatrics 1996;97: 669-675.

29. Ross Products Division, Abbott Laboratories. 2002 Breastfeeding Trends. Report from the Ross Mothers Survey. Abbott Laboratories, Cleveland, OH, 2003.

30. Oregon Public Health Division. PRAMS (Pregnancy Risk Assessment Monitoring System). http://oregon.gov/DHS/ $\mathrm{ph} / \mathrm{pnh} / \mathrm{prams} /$ index.shtml (accessed February 1, 2008).

Address reprint requests to: Kenneth D. Rosenberg, M.D., MPH

Office of Family Health

Oregon Public Health Division

800 NE Oregon Street, Suite 850

Portland, OR 97232

E-mail: ken.d.rosenberg@state.or.us 